\title{
Bianchi type I universe in brane world scenario with non-zero Weyl tensor of the bulk
}

\author{
S. Chaudhuri ${ }^{\mathrm{a}}$ \\ Department of Physics, University of Burdwan, Burdwan 713 104, India
}

Received: 17 July 2017 / Accepted: 28 August 2017 / Published online: 18 September 2017

(C) The Author(s) 2017. This article is an open access publication

\begin{abstract}
In the paper, we present exact solutions of gravitational field equations for an anisotropic brane with a Bianchi type I universe with perfect fluid having nonvanishing Weyl tensor of the bulk. It is assumed that the thermodynamic pressure bears a linear relation with the energy density. For a particular non-zero value of the pressure the solutions are obtained in an exact analytic form with and without the cosmological constant for a Bianchi type I universe. The relevant physical quantities associated with the evolution of the universe are also derived in the two cases.
\end{abstract}

\section{Introduction}

During the past one and a half decade the evolution of the universe in the brane world scenario is a much discussed topic in cosmology. Randall and Sundrum (RS) presented a theory of gravity with an infinite fifth dimension in the presence of a brane [1,2]. RS suggested a brane model in which the standard model particles are confined in a hypersurface called brane with constant negative tension embedded in a higher dimensional space-time called the bulk. The bulk is a space time with negative cosmological constant. Only gravity and other exotic matter can propagate in the bulk [3]. Investigations are going on if our universe might be such a brane-like object. Extensive studies on brane cosmology have been done by many research workers. For a detailed analysis of brane cosmology, to name only a few, one may consult Refs. [4-10].

Campos and Sopuerta [6] assumed a linear barotropic equation of state $p=(\gamma-1) \rho$ on the brane and presented a qualitative analysis of the behaviour of Bianchi type I and $\mathrm{V}$ cosmological models. Anisotropy may grow in intermediate stages during the expansion of the universe. A detailed study of the geometry and dynamics of the brane world has been done by Maartens [10]. An anisotropic Bianchi type I

a e-mail: schaudhuri.phys@gmail.com brane-world in the presence of inflationary scalar fields was considered by Maartens et al. [11]. They showed that the magnitude of the anisotropy parameter of the brane does not affect inflation.

Chen et al. [9] presented exact solutions of the gravitational field equations in the generalized RS model for an anisotropic brane with Bianchi type I and V geometry with perfect fluids and scalar fields as matter sources. They considered the bulk to be conformally flat with vanishing Weyl tensor and showed that the quadratic terms arising in the energy-momentum tensor of the perfect cosmological fluids lead to major changes in the early dynamics of the anisotropic universe as compared to the standard general relativistic case [9].

We shall make an attempt to derive the field equations for an anisotropic brane in the case of a Bianchi type I universe with perfect fluid model having nonvanishing Weyl tensor of the bulk. It is shown that the effect of the inclusion of the energy term for non-zero Weyl tensor of the bulk in the analysis is reflected in the gravitational field equations as an additive term in the parametric expressions compared to the results of Chen et al. [9]. By redefining the initial cosmological time, it is shown that the brane universe evolves from a singular state with volume scale factor equal to zero from time $t=0$. For a particular non-zero value of the pressure $(p)$ (but less than the matter density $(\rho)$ ) and under some restrictions on the constants, the solutions are obtained in an exact analytic form with and without the cosmological constant for a Bianchi type I universe. The evolution of the volume scale factor, expansion scalar, deceleration parameter, shear and the anisotropy are evaluated in both cases.

\section{Field equations}

In this section we discuss the relevant field equations describing the bulk and the brane universe. 
The bulk is assumed to be a 5-dimensional space-time and the brane, embedded in the bulk, has 4 dimensions. In the 5dimensional space-time the brane is assumed to be located at $Y\left(X^{I}\right)=0,(I=0,1,2,3,4)$ The Einstein field equations for the bulk are given by [9]

$$
\begin{aligned}
G_{I J} & =K_{5}^{2} T_{I J}, \\
T_{I J} & =-\Lambda_{5} g_{I J}+\delta(Y)\left[-\lambda g_{I J}+T_{I J}^{\mathrm{Matter}}\right],
\end{aligned}
$$

where $K_{5}^{2}=8 \pi G_{5}$ is the 5-dimensional gravitational coupling constant, $\Lambda_{5}$ is the vacuum energy of the bulk, $\lambda$ is the vacuum energy on the brane and $I, J$ take the values $0,1,2$, 3,4 . The vacuum energy represented by $\Lambda_{5}$ is assumed to be negative and is the only source of the gravitational field [9].

The metric is taken in the form [9]

$\mathrm{d} s^{2}=\left(n_{I} n_{J}+g_{I J}\right) \mathrm{d} x^{I} \mathrm{~d} x^{J}$

where $n_{I} \mathrm{~d} x^{I}=\mathrm{d} \chi$ is the unit normal to the $\chi=$ constant hypersurfaces, $g_{I J}$ is the metric induced on $\chi=$ constant hypersurfaces. The effective 4-dimensional gravitational field equations on the brane take the form [7-9]

$G_{\mu \nu}=-\Lambda g_{\mu \nu}+K_{4}^{2} T_{\mu \nu}+K_{5}^{4} S_{\mu \nu}-E_{\mu \nu}$

where

$S_{\mu \nu}=\frac{1}{12} T T_{\mu \nu}-\frac{1}{4} T_{\mu}^{\alpha} T_{\nu \alpha}+\frac{1}{24} g_{\mu \nu}\left(3 T^{\alpha \beta} T_{\alpha \beta}-T^{2}\right)$,

$\Lambda=\frac{1}{2} K_{5}^{2}\left(\Lambda_{5}+\frac{1}{6} K_{5}^{2} \lambda^{2}\right)$,

$K_{4}^{2}=\frac{1}{6} \lambda K_{5}^{4}$,

$E_{I J}=C_{I A J B} n^{A} n^{B}$,

$C_{I A J B}$ is the 5-dimensional Weyl tensor in the bulk; it is traceless $[7,10] . E_{\mu \nu}$ is the projection of the bulk Weyl tensor on the brane. This represents the nonlocal effects on the brane from the free gravitational field in the bulk. $T_{\mu \nu}$ is the matter energy-momentum tensor on the brane and $T=T_{\mu}^{\mu}$ is the trace of the energy-momentum tensor. The components of $T_{\mu \nu}$ are [9]

$T_{o}^{o}=-\rho, \quad T_{1}^{1}=T_{2}^{2}=T_{3}^{3}=p$.

We now introduce the directional Hubble parameters $\left(H_{i}\right)$ and the mean Hubble parameter $(H)$ :

$H_{i}=\frac{\dot{a}_{i}}{a_{i}}, \quad i=1,2,3$,

$H=\frac{1}{3} \sum_{i=1}^{3} H_{i}$, where $a_{i}$ are given by

$V=\prod_{i=1}^{3} a_{i}$

$V$ is known as volume scale factor. A dot represents differentiation with respect to time.

The relevant quantities of physical interest such as the expansion scalar $(\theta)$, mean anisotropy parameter $(A)$, shear scalar $\left(\sigma^{2}\right)$ and the deceleration parameter $(q)$ are defined in terms of the Hubble parameters $\left(H_{i}\right.$ and $\left.H\right)$ by [9]

$\theta=3 H$

$A=\frac{1}{3} \sum_{i=1}^{3}\left(\frac{\Delta H_{i}}{H}\right)^{2}$,

$\sigma^{2}=\frac{3}{2} A H^{2}$

$q=\frac{\mathrm{d}}{\mathrm{d} t}\left(\frac{1}{H}\right)-1$,

where

$\Delta H_{i}=H_{i}-H, \quad i=1,2,3$.

For a Bianchi type I universe, the metric is given by

$\mathrm{d} s^{2}=-\mathrm{d} t^{2}+a_{1}^{2}(t) \mathrm{d} x^{2}+a_{2}^{2}(t) \mathrm{d} y^{2}+a_{3}^{2}(t) \mathrm{d} z^{2}$.

From Eqs. (7)-(9) the Hubble parameter is expressed as

$H=\frac{\dot{V}}{3 V}$.

We consider that the bulk has non-zero Weyl tensor and since it is traceless the values of $E_{\mu}^{\mu}$ are assumed to be of the form [10]

$E_{o}^{o}=-\frac{6 U}{K_{4}^{2} \lambda}, \quad E_{1}^{1}=E_{2}^{2}=E_{3}^{3}=\frac{2 U}{K_{4}^{2} \lambda}$,

where the function $U$ satisfies the relation [10]

$\dot{U}+4 H U=0$.

From Eqs. (16) and (18) one obtains

$U=\alpha V^{-4 / 3}$

$\alpha$ being a constant.

We further assume that the pressure $p$ bears a linear barotropic relation with the energy density $\rho$ given by [9]

$p=(\gamma-1) \rho$

where $\gamma$ is a constant; it can take values $1 \leq \gamma \leq 2$. 
With the help of Eqs. (7)-(9), the gravitational field equations on the brane are obtained:

$$
\begin{aligned}
3 \dot{H}+\sum_{i=1}^{3} H_{i}^{2}= & \Lambda-\frac{(3 \gamma-2)}{2} K_{4}^{2} \rho \\
& -\frac{(3 \gamma-1)}{12} K_{5}^{4} \rho^{2}+\frac{6 \alpha}{K_{4}^{2} \lambda} V^{-4 / 3}, \\
\frac{1}{V} \frac{\mathrm{d}}{\mathrm{d} t}\left(V H_{i}\right)= & \Lambda-\frac{(\gamma-2)}{2} K_{4}^{2} \rho \\
& -\frac{(\gamma-1)}{12} K_{5}^{4} \rho^{2}-\frac{2 \alpha}{K_{4}^{2} \lambda} V^{-4 / 3},
\end{aligned}
$$

and

$\dot{\rho}=3 \gamma H \rho$.

The last term on the right hand side of Eqs. (21) and (22) represents the effect of Weyl tensor of the bulk on the brane. If one substitutes $\alpha=0$, one recovers the corresponding equations of Chen et al. (Eqs. (16) and (17) of Ref. [9]). From Eq. (22) we obtain

$$
\begin{aligned}
\frac{1}{V} \frac{\mathrm{d}}{\mathrm{d} t}(V H)= & \Lambda-\frac{(\gamma-2)}{2} K_{4}^{2} \rho-\frac{(\gamma-1)}{12} K_{5}^{4} \rho^{2} \\
& -\frac{2 \alpha}{K_{4}^{2} \lambda} V^{-4 / 3} .
\end{aligned}
$$

The relation between the directional Hubble parameters $\left(H_{i}\right)$ and mean Hubble parameter $(H)$ is obtained by subtracting Eq. (24) from (22) and is given by

$H_{i}-H=\frac{C_{i}}{V}$

where $C_{i}$ is a constant with $i=1,2,3$.

\section{Solutions of brane universe}

We now find the solutions of the brane universe embedded in a 5-D bulk in general.

From Eq. (23), the energy density is obtained:

$\rho=\rho_{o} V^{-\gamma}, \quad \rho=$ constant $>0$.

The dynamical equation for the anisotropic brane world with non-zero Weyl tensor of the bulk is obtained from Eq. (22) and is given by

$$
\begin{aligned}
\ddot{V}= & 3 \Lambda V-\frac{3(\gamma-2)}{2} K_{4}^{2} \rho_{o} V^{1-\gamma} \\
& -\frac{(\gamma-1)}{4} K_{5}^{4} \rho_{o}^{2} V^{1-2 \gamma}-\frac{6 \alpha}{K_{4}^{2} \lambda} V^{-1 / 3} .
\end{aligned}
$$

If one redefines the initial cosmological time in such a manner that while integrating Eq. (27) one may set the constant of integration equal to zero, then from Eq. (27) one obtains

$$
t-t_{o}=\int \frac{\mathrm{d} V}{\left[3 \Lambda V^{2}+3 K_{4}^{2} \rho_{o} V^{2-\gamma}+\frac{1}{4} K_{5}^{4} \rho_{o}^{2} V^{2-2 \gamma}-\frac{18 \alpha}{K_{4}^{2} \lambda} V^{2 / 3}\right]^{\frac{1}{2}}} .
$$

Using Eqs. (27) and (28), the expressions for the expansion scalar $(\theta)$, anisotropy $(A)$, shear $\left(\sigma^{2}\right)$ and deceleration parameter $(q)$ are obtained from Eqs. (10)-(13):

$\theta=\left[3 \Lambda+3 K_{4}^{2} \rho_{o} V^{-\gamma}+\frac{1}{4} K_{5}^{4} \rho_{o}^{2} V^{-2 \gamma}-\frac{18 \alpha}{K_{4}^{2} \lambda} V^{-4 / 3}\right]^{1 / 2}$,

$A=3 C^{2}\left[3 \Lambda V^{2}+3 K_{4}^{2} \rho_{o} V^{2-\gamma}+\frac{1}{4} K_{5}^{4} \rho_{o}^{2} V^{2-2 \gamma}-\frac{18 \alpha}{K_{4}^{2} \lambda} V^{2 / 3}\right]^{-1}$,

$\sigma^{2}=\frac{C^{2}}{2 V^{2}}$,

$q=2-\frac{36 \Lambda V^{2}+18(2-\gamma) K_{4}^{2} \rho_{o} V^{2-\gamma}+3(1-\gamma) K_{5}^{4} \rho_{o}^{2} V^{2-2 \gamma}-\frac{72 \alpha}{K_{\lambda}^{2} \lambda} V^{2 / 3}}{12 \Lambda V^{2}+12 K_{4}^{2} \rho_{o} V^{2-\gamma}+K_{5}^{4} \rho_{o}^{2} V^{2(1-\gamma)}-\frac{72 \alpha}{K_{4}^{2} \lambda} V^{2 / 3}}$,

where

$C^{2}=C_{1}^{2}+C_{2}^{2}+C_{3}^{2}$.

We now consider the solutions presented in Eqs. (27)-(32) for a particular case with $\gamma=\frac{4}{3}$ for which the pressure $(p)$ and energy density $(\rho)$ are related by $p=\frac{1}{3} \rho$.

In this case, we have from Eq. (28),

$t-t_{o}=\int\left[3 \Lambda V^{8 / 3}+\eta V^{4 / 3}+\beta\right]^{-1 / 2} V^{1 / 3} \mathrm{~d} V$,

where

$\eta=3 K_{4}^{2} \rho_{o}-\frac{18 \alpha}{K_{4}^{2} \lambda}, \quad \beta=\frac{1}{4} K_{5}^{4} \rho_{o}^{2}$.

From Eqs. (10)-(14) and (34), the following results are obtained:

$V(t)=\left\{2 \sqrt{2}(3 \Lambda)^{3 / 4}\right\}^{-1} e^{-s} N^{3 / 4}$,

$t=t_{o}+\frac{3}{4} \frac{1}{\sqrt{3 \Lambda}} \ln (\eta+2 \sqrt{3 \Lambda \beta})$,

$\theta=\sqrt{3 \Lambda} N^{-1} D$,

$A=8 \sqrt{3 \Lambda} C^{2} e^{2 s} D^{-2} N^{1 / 2}$,

$\sigma^{2}=4(3 \Lambda)^{3 / 2} C^{2} e^{2 s} N^{-3 / 2}$, 
$q=8 e^{\frac{4}{3} s} D^{-2}\left[\eta\left(e^{\frac{4}{3} s}-\eta\right)^{2}+12 \Lambda \beta\left(2 e^{\frac{4}{3} s}-\eta\right)\right]-1$,

where

$s=\sqrt{3 \Lambda}\left(t-t_{o}\right)$,

$N=\left(e^{\frac{4}{3} s}-\eta\right)^{2}-12 \Lambda \beta$,

$D=e^{\frac{8}{3} s}-\eta^{2}+12 \Lambda \beta$.

If the initial time $t_{0}$ is redefined according to

$e^{-\frac{4}{3} \sqrt{3 \Lambda} t_{o}}=\eta+2 \sqrt{3 \Lambda \beta}$

the evolution of the brane universe starts from a singular state,

$V_{o}=V(t=0)=0$ at time $t=0$.

If we set the cosmological constant $\Lambda=0$, the expressions for $V, \theta, A, \sigma^{2}$ and $q$ on the brane universe are obtained:

$V(t)=(9 \eta)^{-3 / 4} P^{3 / 4}$,

$\theta=6 \eta^{2}\left(t-t_{o}\right) P^{-1}$,

$A=\left(\frac{9}{4}\right) C^{2} \eta^{-5 / 2}\left(t-t_{o}\right)^{-2} P^{\frac{1}{2}}$,

$\sigma^{2}=\left(\frac{27}{2}\right) C^{2} \eta^{3 / 2} P^{-3 / 2}$,

$q=1+\left(\frac{9}{2}\right) \beta\left[\eta\left(t-t_{o}\right)\right]^{-2}$,

$P=4 \eta^{2}\left(t-t_{0}\right)^{2}-9 \beta$.

From Eqs. (47) and (52) one finds that $V_{0}=V(t=0)=$ 0 , if the initial time is redefined by

$t_{0}^{2}=\frac{9 \beta}{4 \eta^{2}}$

The evolution of the brane universe with and without the cosmological constant is thus described in Eqs. (34)-(52).

\section{Conclusions}

It is evident from Eq. (46) that the brane universe evolves from a singular state $V=0$, from time $t=0$ when the initial time is defined according to Eq. (45). At $t=0$, the anisotropy vanishes and the expansion scalar $(\theta)$ becomes infinitely large. In the early stages of evolution when $t$ is small the volume scale factor and the anisotropy increase with time.
When $t \rightarrow \infty$, the volume scale factor $V$ becomes infinitely large and the anisotropy decreases with time. The anisotropy thus starting from value zero increases to a maximum in some intermediate stages of evolution and then again decreases with the passage of time.

For a universe with cosmological constant $\Lambda=0$, the evolution of the brane universe starts from $t=0$ from a singular state $V=0$ when the initial cosmological time is redefined by $t_{o}^{2}=\frac{9 \beta}{4 \eta^{2}}$ (Eq. (53); $\beta$ and $\eta$ are defined in Eq. (35)). At $t=0$, the anisotropy $A=0$, the expansion scalar becomes infinite and the deceleration parameter $q$ assumes a constant value, $q=3$. When $t$ increases from its zero value, the volume scale factor also increases. For large $t$, the anisotropy varies as $\frac{1}{t}$ and as $t \rightarrow \infty$, the volume scale factor goes to infinity, the anisotropy $A \rightarrow 0$, and the deceleration parameter q becomes unity.

The effect of the Weyl tensor of the bulk on the brane is reflected in the expression for $\eta$ (see Eq. (35)). It appears from Eqs. (36) and (39) (for $\Lambda \neq 0$ ) and Eqs. (47) and (49) (for $\Lambda=0$ ) that the increase in volume scale factor and the decrease in anisotropy with time become slower in the presence of the Weyl tensor of the bulk.

Acknowledgements The author acknowledges the University Grants Commission, India for financial support (vide Research Project no. F. PSW- 07/ 06 - 07 (ERO)) and Inter-University Centre for Astronomy and Astrophysics, Pune, India. The author is grateful to Prof. S. Chakraborty, Jadavpur University, for drawing attention to the problem and for many useful discussions thereafter.

Open Access This article is distributed under the terms of the Creative Commons Attribution 4.0 International License (http://creativecomm ons.org/licenses/by/4.0/), which permits unrestricted use, distribution, and reproduction in any medium, provided you give appropriate credit to the original author(s) and the source, provide a link to the Creative Commons license, and indicate if changes were made. Funded by $\mathrm{SCOAP}^{3}$.

\section{References}

1. L. Randall, R. Sundrum, Phys. Rev. Lett. 83, 3370 (1999)

2. L. Randall, R. Sundrum, Phys. Rev. Lett. 83, 4690 (1999)

3. P. Brax, C. Van de Bruck, Class. Quantum Gravity 20, R201 (2003)

4. C.-M. Chen, T. Harko, M.K. Mak, Phys. Rev. D 61, 104017 (2000)

5. R. Maartens, Phys. Rev. D 62, 084032 (2000)

6. A. Campos, C.F. Sopuerta, Phys. Rev. D 63, 104012 (2001)

7. T. Shiromizu, K. Maeda, M. Sasaki, Phys. Rev. D 62, 024012 (2000)

8. M. Sasaki, T. Shiromizu, K. Maeda, Phys. Rev. D 62, 024008 (2000)

9. C.-M. Chen, T. Harko, M.K. Mak, Phys. Rev. D 64, 044013 (2001)

10. R. Maartens. arXiv:gr-qc/0101059 [V2] (2001)

11. R. Maartens, V. Sahni, T.D. Saini, Phys. Rev. D 63, 063509 (2001) 